\title{
Model-based iterative reconstruction in paediatric head computed tomography: a pilot study on dose reduction in children
}

\author{
Pardeep K. Atri ${ }^{A, B, C, D, E, F, F}$, Kushaljit S. Sodhi ${ }^{A, B, C, D, E, F}$, Anmol Bhatia ${ }^{A, B, C, D, E, F}$, Akshay K. Saxena ${ }^{A, C, D, E, E}$, \\ Niranjan Khandelwal ${ }^{A, B, D, E}$, Pratibha Singhi ${ }^{A, B, D, E}$ \\ Post Graduate Institute of Medical Education \& Research (PGIMER), Chandigarh, India
}

\begin{abstract}
Purpose: To evaluate the potential of model-based iterative reconstruction (MBIR) on dose reduction and image quality in children undergoing computed tomography (CT) head examinations.

Material and methods: This prospective study was approved by the institutional ethics committee. A total of 88 children (age range of 5 to 16 years) with a history of seizures underwent contrast-enhanced CT scan. Forty-one children underwent CT study according to the MBIR technique, while 47 children underwent CT of the head with the nonMBIR protocol. Images were reviewed by 2 blinded paediatric radiologists in a random order. Mean dose-length product, CT dose index (CTDI) volume, and mean effective dose were recorded for both groups. Image quality, image noise, and diagnostic acceptability of 2 image sets were also recorded.

Results: In the MBIR group, the mean dose-length product was reduced by $79.8 \%$; the mean CTDI volume was reduced by $88.5 \%$, while the mean effective dose was reduced by $81 \%$ when compared to the non-MBIR group. No significant difference was seen in diagnostic acceptability, image noise, and image quality between the 2 groups. Conclusions: MBIR technique is highly effective in reducing radiation dose in paediatric head CT examinations without any significant difference in image quality, image noise, and diagnostic acceptability.
\end{abstract}

Key words: CT, children, head, iterative reconstruction, radiation dose.

\section{Introduction}

Computed tomography (CT) is a fast, low-cost, and widely available diagnostic imaging modality. There is increasing concern about radiation-related side effects, especially in children [1]. Repeated CT examinations cause cumulative exposure to ionizing radiation in children, rendering them vulnerable to malignancies later in life, which are actually preventable. The children also have relatively fast-growing cells, and therefore they are inherently more likely to develop malignancies related to radiation exposure [1].
No other radiological investigation has greater radiation risk than that associated with CT exams. Due to the concerns associated with exposure to ionizing radiation, strategies are employed to minimize the radiation dose received during $\mathrm{CT}$ examinations, but not at the cost of compromising the quality of imaging and diagnostic results. The ALARA principle should be applied rigorously in children [1-4]. It is well documented that with correct justification, optimisation, and dose limitation, radiation risks can largely be reduced, if not totally eliminated. Certainly, in children, much more effort is required to guarantee judicious use of CT scans. In recent times, different

Correspondence address:

Dr. Anmol Bhatia, Post Graduate Institute of Medical Education \& Research (PGIMER), Madhya Marg, Sector 12, Chandigarh, 160012, India,

e-mail: anmol_bhatia26@yahoo.co.in

Authors' contribution:

A Study design · B Data collection · C Statistical analysis · D Data interpretation · E Manuscript preparation · F Literature search · G Funds collection 
software and methods for dose reduction have been developed by CT manufacturers [5-7].

$\mathrm{X}$-ray tube current modulation and $\mathrm{kVp}$ optimization have been tried, with some success, for the reduction of the radiation dose, but these have their limitations and may compromise the image quality (because noise is increased) as well as the diagnostic value. Furthermore, noise reduction mathematical algorithms can be applied, which further reduces the radiation dose and simultaneously maintains the image quality by reducing the noise [5-7].

Filtered back projection (FBP) was the earliest of the noise reduction mathematical algorithms used in the image reconstruction. The iterative reconstruction (IR) technique has been a recent advancement in this approach. Due to the simple mathematical computation requirement, analytical reconstruction algorithms like FBP have been used to produce CT images, instead of IR algorithms $[8,9]$. Over the past few years, several IR algorithms have emerged in clinical CT applications. In recent years, model-based iterative reconstruction (MBIR), which is also known as a pure IR algorithm, has been shown to improve the quality of the images significantly, reducing noise and artifacts $[9,10]$. It also improves the spatial resolution. Some studies have been published to evaluate the effectiveness of MBIR and have shown promising results [11-14]. CT head is one of the most common CT examinations performed in children. The present prospective study was conducted to evaluate the potential of MBIR technique on dose reduction and image quality in children undergoing CT head examinations.

\section{Material and methods}

This was a prospective research study conducted over a period of 12 months. Our study was approved by the ethics committee of our institute. Patient confidentiality was maintained in accordance with Health Insurance Portability and Accountability Act guidelines (HIPAA). Informed written consent was taken from the parents/ guardians of all the children. Assent was also obtained from the children aged 7 years and above prior to enrolment.

Eighty-eight consecutive children referred for contrast enhanced CT of the head were enrolled in this study.

Inclusion criteria:

- age: 5-16 years,

- history of seizures (recent or follow-up).
Exclusion criteria:

- children outside the age group, i.e. $<5$ years and $>16$ years,

- parents/guardians/children not consenting for the study.

The children were randomly assigned to MBIR and non-MBIR (based on FBP) groups.

\section{Computed tomography paediatric head image acquisition}

CT head examinations for the non-MBIR group were acquired on a Toshiba aquilion ${ }^{\mathrm{TM}}, 64$-slice CT scanner, while for the MBIR group the images were acquired on a Philips Brilliance iCT 256-slice CT scanner using MBIR technology. The protocols for both the groups are summarized in Table 1. The images from both the study groups were transferred to a common workstation for analysis.

\section{Assessment of image-quality parameters}

The scale for the assessment of image quality was based on a study done by Kilic et al. [15].

(i) Objective (quantitative) assessment:

Image noise was evaluated in air, cerebrospinal fluid (CSF), and white matter (WM). A $60 \mathrm{~mm}^{2}$ ROI was placed in the air (Figure 1A) within $1 \mathrm{~cm}$ of the scalp, at a place free from artefacts, and measurements were taken in 3 different regions (at the level of posterior fossa). Image noise was measured in CSF (Figure 1B) by keeping an ROI with an area of $>15 \mathrm{~mm}^{2}$ placed in lateral ventricles, or suitable CSF spaces if the measurement could not be performed in a lateral ventricle. The third ROI was measured in WM (Figure 1C) at the level of centrum semiovale placed in areas free of disease and distant from pathological sites.

Contrast-to-noise ratio (CNR) was calculated by taking the means of 3 ROIs measured in grey matter (GM) (Figure 1C) and 3 in the WM measured at the level of centrum semiovale avoiding partial-volume effects. CTn and SD values were recorded and thereafter averaged for each part. $\mathrm{CNR}=$ mean GM CTn - mean WM CTn/

$\left[(\text { mean SD in } \mathrm{GM})^{2}+(\text { mean SD in } \mathrm{WM})^{2}\right]^{1 / 2}$.

Signal-to-noise ratio (SNR) was calculated by first taking means of the CTn and SD in 3 ROIs in WM at the level of the centrum semiovale.

SNR WM = mean CTn/mean SD

ii) Subjective (qualitative) assessment:

Qualitative assessment was based on subjective image noise, image sharpness, diagnostic acceptability, and artefacts. The analysis was done by 2 radiologists indepen-

Table 1. Table summarizing the computed tomography protocols of non-model-based iterative reconstruction (MBIR) and MBIR groups

\begin{tabular}{|l|c|c|c|c|c|c|c|c|c|}
\hline S. & Age group & \multicolumn{4}{|c|}{ Non-MBIR group } & \multicolumn{4}{c|}{ MBIR group } \\
\cline { 3 - 11 } No. & (in years) & Mode & $\mathbf{k V p}$ & $\mathbf{m A}$ & Rotation time in seconds & Mode & $\mathbf{k V p}$ & $\mathrm{mA}$ & Rotation time in seconds \\
\hline 1 & $5-10$ & Axial & 120 & 180 & 1 & Axial & 120 & 200 & 0.5 \\
\hline 2 & $10-16$ & Axial & 120 & 260 & 1 & Axial & 120 & 300 & 0.5 \\
\hline
\end{tabular}



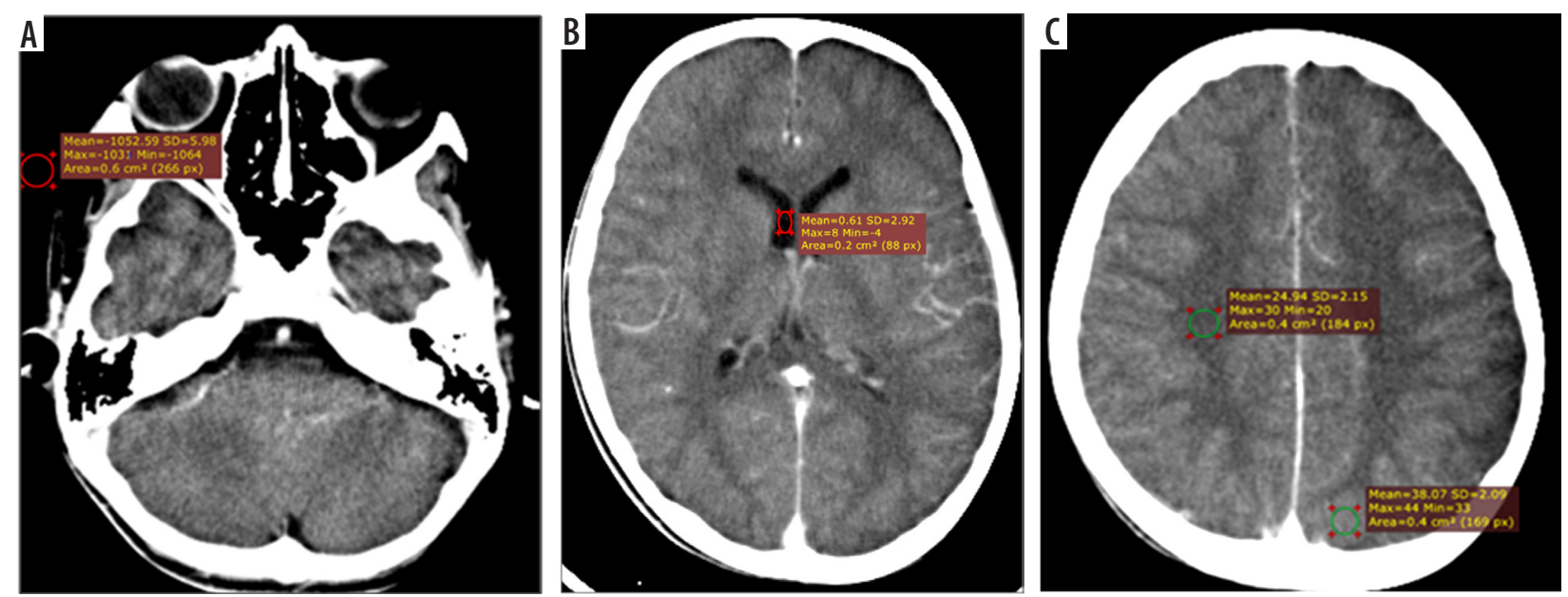

Figure 1. Axial computed tomography images showing region of interest (ROI) placed in air (A), lateral ventricle (B), and grey matter (C)

dently (having 14 and 7 years of experience in paediatric radiology), after anonymizing non-MBIR and MBIR studies (blinded to all clinical, technical, and personal medical information). The images were analysed in a randomized order. A window level of 40-60 HU and window width of 60-90 HU was maintained for all the images. Each subjective parameter of image analysis was assessed on a scale of 4 to 5 points described as follows:

Noise: 1 - least, 2 - optimum, 3 - above average, 4 - too much noise, no information provided.

Sharpness: 1 - above average sharpness, 2 - average sharpness, 3 - below average sharpness, 4 - not sufficient for diagnosis, 5 - structures cannot be identified.

GM and WM differentiation, basal ganglia, pons, and margins of the sulci/gyri were used to assess the sharpness.

Diagnostic acceptability: 1 - fully acceptable, 2 - probably-acceptable, 3 - acceptable but under limited conditions, 4 - not acceptable.

Artefacts: 1 - none, 2 - minor, 3 - major but interpretable, 4 - image interpretation not possible.

\section{Radiation dose estimates}

The effective dose-mSv (ED) was calculated by multiplying the dose length product with an age-wise value of the conversion factor for $\mathrm{CT}$ head examinations according to ICRP Publication 60 Recommendations [16].

We used 0.0031 as a conversion factor for the age group 5-10 years and 0.0023 for $10-16$ years in our study.

\section{Statistical analysis}

All the statistical analysis was done using Statistical Package for the Social Sciences (Version 22.0). An unpaired Student $t$-test was used for continuous variables. Wilcoxon rank sum (Mann-Whitney $U$ ) test was used for categorical variables. The kappa test and $\chi^{2}$ test were applied to assess the subjective parameters determined by the 2 radiologists. All the statistical tests were 2 -sided, performed at a significance level of $\alpha=0.05$.

\section{Results}

\section{Age distribution}

There were 41 children in the MBIR group, of whom 22 were 5-10 years old and 19 were $10-16$ years old. There were 47 children in the MBIR group, of whom 33 were 5-10 years old and 14 were 10-16 years old. The difference in sample size in both groups was not statistically significant $(p>0.05)$.

\section{Radiation dose analysis}

The radiation exposure in terms of effective dose (ED), dose length product (DLP), and CT dose index volume $\left(\mathrm{CTDI}_{\mathrm{vol}}\right)$ showed a significant reduction in the MBIR group compared to the non-MBIR group.

There was an overall reduction in mean effective dose in the MBIR group by $81 \%$. The reduction was similar in both the age groups ( $81.6 \%$ in $5-10$ years, $80.3 \%$ in $10-16$ years).

The overall reduction in mean DLP was $79.8 \%$. The reduction in DLP was also similar in both the age groups ( $81.6 \%$ in $5-10$ years, $80.3 \%$ in $10-16$ years).

The mean CT dose index volume was also reduced in the MBIR group, and the overall reduction was $88.5 \%$. The age-wise reduction in $\mathrm{CTDI}_{\mathrm{vol}}$ in both the age groups was similar ( $89.6 \%$ in $5-10$ years, $88.8 \%$ in $10-16$ years).

All the reductions were statistically significant $(p<0.05)$.

Table 2 summarizes the mean ED, DLP, and CTDI in the MBIR and non-MBIR groups. Table 3 summarizes the age-wise comparison of radiation dose parameters between the MBIR and non-MBIR groups.

\section{Objective image analysis}

Table 4 summarizes objective image analysis in the 2 groups. No statistically significant difference was seen with regards to measured noise in WM and CNR. The overall SNR was higher in the non-MBIR group. The overall measured noise 


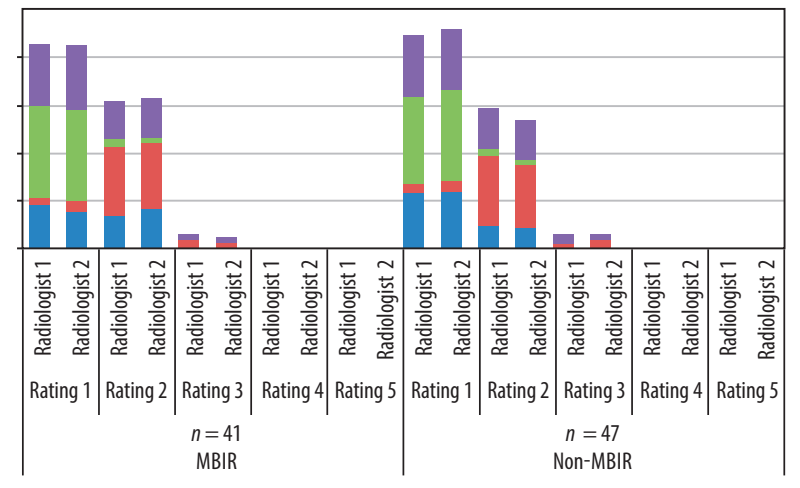

Subjective image noise \% — Subjective image sharpness - Diagnostic acceptability \% — Artefacts

Figure 2. Bar chart comparing the subjective assessment for image quality done by the 2 radiologists. There was a high degree of inter-observer agreement seen for all the parameters observed. None of the parameters was ranked 4 or 5

in air and CSF was lower in the MBIR group. The difference in measured noise in CSF in the 2 study groups in the 10-16-years age group was not statistically significant $(p>0.05)$.

\section{Subjective image analysis}

There was very good interobserver strength of agreement for the subjective image noise $(\mathrm{K}-0.833, p<0.05$, Table 5$)$, subjective image sharpness (K-0.824, $p<0.05$, Table 6$)$, diagnostic acceptability (K-0.822, $p<0.05$, Table 7$)$, and for artefacts $(\mathrm{K}-0.802, p<0.05$, Table 8$)$.

The subjective assessments of image quality by the 2 radiologists were compared and are summarized in Figure 2. The differences between inter-observer ratings were not statistically different between the MBIR and non-MBIR groups except for the subjective image noise assessment by both the radiologists $(p=0.040)$. None of the MBIR/nonMBIR was rated as 4 or 5 for any of the parameters.

\section{Discussion}

In the present study, the potential of MBIR to reduce radiation-related dose parameters (ED, DLP, and CTDI ${ }_{\text {vol }}$ ) compared to conventional filtered back projections was assessed, and we found an overall reduction of $81 \%$ in

Table 2. Table summarizing the mean effective dose (ED), dose length product (DLP), and computed tomography dose index volume (CTDI ${ }_{\text {vol }}$ ) in the model-based iterative reconstruction (MBIR) and non-MBIR groups

\begin{tabular}{|l|c|c|c|c|c|}
\hline \multirow{2}{*}{} & \multicolumn{2}{|c|}{ MBIR group } & \multicolumn{2}{c|}{ Non-MBIR group } & \multirow{2}{*}{$p$-value } \\
\cline { 2 - 5 } & Mean & SD & Mean & SD & \\
\hline CTDI $_{\text {vol }}(\mathrm{mGy})$ & 9.85 & 2.02 & 85.86 & 17.00 & 0.0001 \\
\hline DLP $(\mathrm{mGy} c \mathrm{~cm})$ & 132.59 & 31.03 & 656.86 & 145.12 & 0.0001 \\
\hline ED $(\mathrm{mSv})$ & 0.35 & 0.04 & 1.84 & 0.28 & 0.0001 \\
\hline
\end{tabular}

SD - standard deviation mean ED, $79.8 \%$ in DLP, and $88.5 \%$ in CTDI $_{\text {vol }}$. There was overall $81 \%$ reduction in mean $\mathrm{ED}, 79.8 \%$ in mean DLP, and $89 \%$ in mean CTDI $_{\text {vol }}$ in the MBIR group in our study. This is consistent with studies performed previously in adult body imaging [16]. No significant differences in the reduction in radiation dose parameters were seen in the 2 different age groups in the present study. No statistically significant difference was found in the image quality between the 2 groups in our study. Although the SNR was higher in the non-MBIR group, the overall noise in air and CSF was lower in the MBIR group; these findings are not significant because there was no effect on diagnostic acceptability. In addition, subjective assessment of image quality was similar in both the study groups and had a high inter-observer agreement. Lower measured noise in MBIR has been documented and published in previous studies [16], but no significant differences were documented in the results of the majority of subjectively assessed image quality parameters and in diagnostic acceptability.

Multiple techniques have been incorporated in an attempt to reduce the radiation dose while performing head CTs, which include but are not limited to reducing mAs, shields, angling the gantry to exclude the orbits, and automatic tube current modulation. Reducing mAs to reduce radiation dose goes hand in hand with increasing noise, thus leading to reduced image quality and interpretability. But if we reduce the noise by creating specific adaptive filters, we get an opportunity to reduce the $\mathrm{mA}$ and hence the radiation dose. MBIR reduces the radiation dose and at the same time maintains the image quality. There have been encouraging results with MBIR in chest and body imaging in the adult population [17]. However, it has limited validation in the paediatric population. We have evaluated and tried to validate the role of MBIR in the paediatric population in the present study.

According to the American College of Rheumatology (ACR) paediatric CT accreditation standards, the maximum acceptable phantom CTDI ${ }_{\text {vol }}$ dose for a paediatric head CT examination is $40 \mathrm{mGy}$ (for a 1-year-old child). Furthermore, for paediatric head CT examinations the estimated mean reported CTDI values have been reported to be $27.3 \mathrm{mGy}$ (95\% CI, 24.4-30.1 mGy), with a DLP of 390.9 mGy cm [18]. The overall mean CTDI ${ }_{\text {vol }}$ and DLP in our study were $9.85 \mathrm{mGy}$ and $132.59 \mathrm{mGy} \mathrm{cm}$, respectively. Hence, a significant reduction can be made in radiation doses by employing MBIR.

In a study done by Meivelle et al. [19], children with cystic fibrosis were studied with standard CT and moderately reduced-dose CT plus a minimum-dose CT. Standard CT images were reconstructed with the FBP technique, while low-dose CT images were reconstructed with both FBP and VEO (MBIR). The images were subjected to qualitative assessment by radiologists as well as objective assessment. Compared to FBP images, VEO images showed significantly lower SD $(p<0.001)$ and higher SNR $(p<0.05)$. 
Table 3. Age-wise comparison of radiation dose parameters between model-based iterative reconstruction (MBIR) and non-MBIR group

\begin{tabular}{|c|c|c|c|c|c|}
\hline & $n$ & MBIR group & $n$ & Non-MBIR group & $p$-value \\
\hline \multicolumn{6}{|c|}{$\mathrm{CTDI}_{\text {vol' }}$ mean $\pm \mathrm{SD}(\mathrm{mGy} \mathrm{cm})$} \\
\hline 5-10 years & 22 & $8.00 \pm 0.00$ & 33 & $76.66 \pm 7.62$ & 0.0001 \\
\hline $10-16$ years & 19 & $12.00 \pm 0.00$ & 14 & $107.52 \pm 12.61$ & 0.0001 \\
\hline \multicolumn{6}{|c|}{$\mathrm{DLP}$, mean $\pm \mathrm{SD}(\mathrm{mGy} \mathrm{cm})$} \\
\hline 5-10 years & 22 & $108.38 \pm 8.76$ & 33 & $590.86 \pm 85.9$ & 0.0001 \\
\hline $10-16$ years & 19 & $160.10 \pm 23.69$ & 14 & $812.42 \pm 138.54$ & 0.0001 \\
\hline \multicolumn{6}{|c|}{$\mathrm{ED}, \underline{\text { mean } \pm \mathrm{SD}(\mathrm{mSv})}$} \\
\hline 5-10 years & 22 & $0.33 \pm 0.02$ & 33 & $1.83 \pm 0.26$ & 0.0001 \\
\hline $10-16$ years & 19 & $0.36 \pm 0.05$ & 14 & $1.86 \pm 0.31$ & 0.0001 \\
\hline
\end{tabular}

$\mathrm{CTDI}_{\mathrm{vol}}$ - computed tomography dose index volume, DLP - dose length product, ED - effective dose

Table 4. Table summarizing the mean measurement of noise (objective analysis)

\begin{tabular}{|l|c|c|c|}
\hline \multicolumn{1}{|c|}{} & MBIR group $(n=41)$ & Non-MBIR group $(n=47)$ & $p$-value \\
\hline Measured noise in WM & $3.39 \pm 0.36$ & $3.38 \pm 0.64$ & 0.974 \\
\hline Measured noise in CSF & $3.67 \pm 0.72$ & $4.59 \pm 1.69$ & 0.001 \\
\hline Measured noise in air & $3.52 \pm 0.48$ & $8.52 \pm 2.59$ & 0.001 \\
\hline CNR & $7.63 \pm 3.14$ & $7.15 \pm 4.38$ & 0.558 \\
\hline SNR & $8.57 \pm 0.66$ & $9.53 \pm 1.17$ & 0.001 \\
\hline
\end{tabular}

MBIR - model-based iterative reconstruction, WM - white matter, CSF - cerebrospinal fluid, CNR - contrast-to-noise ratio, SNR - signal-to-noise ratio

Table 5. Comparison of the subjective assessment of image noise done by Radiologist 1 and Radiologist 2

\begin{tabular}{|c|c|c|c|c|c|c|c|}
\hline \multicolumn{3}{|l|}{ Group } & \multicolumn{2}{|c|}{ Subjective image noise - R2 } & \multirow{2}{*}{$\begin{array}{l}\text { Total } \\
23 \\
\end{array}$} & \multirow{3}{*}{$\begin{array}{c}\text { K-value } \\
0.854\end{array}$} & \multirow{2}{*}{$\begin{array}{l}p \text {-value } \\
0.0001\end{array}$} \\
\hline MBIR & Subjective image & $1-$ no noise & $\begin{array}{c}1-\text { No noise } \\
20 \\
\end{array}$ & $\begin{array}{c}2-\text { Optimum noise } \\
3\end{array}$ & & & \\
\hline & noise - R1 & 2-optimum noise & & 18 & 18 & & \\
\hline \multirow[t]{2}{*}{ Non-MBIR } & \multirow{2}{*}{$\begin{array}{l}\text { Subjective image } \\
\text { noise - R1 }\end{array}$} & $1-$ no noise & 30 & 1 & 31 & \multirow[t]{2}{*}{0.805} & \multirow[t]{2}{*}{0.0001} \\
\hline & & 2-optimum noise & 3 & 13 & 16 & & \\
\hline
\end{tabular}

MBIR - model-based iterative reconstruction

Table 6. Comparison of the subjective assessment for image sharpness done by Radiologist 1 and Radiologist 2

\begin{tabular}{|c|c|c|c|c|c|c|c|c|}
\hline \multirow{2}{*}{\multicolumn{2}{|c|}{ Group }} & \multirow{3}{*}{$\begin{array}{l}\text { Rating } \\
\text { 1- above average sharpness } \\
\end{array}$} & \multirow{2}{*}{\multicolumn{3}{|c|}{ Subjective Image sharpness- $\mathbf{R} 2$}} & \multirow{2}{*}{\begin{tabular}{|c|} 
Total \\
\\
3 \\
\end{tabular}} & \multirow{4}{*}{$\begin{array}{c}\kappa \text {-value } \\
0.834\end{array}$} & \multirow{2}{*}{$\begin{array}{l}p \text {-value } \\
0.0001\end{array}$} \\
\hline & & & & & & & & \\
\hline MBIR & Subjective & & \multirow{2}{*}{$\begin{array}{c}\begin{array}{c}\text { - Above average } \\
\text { sharpness }\end{array} \\
3 \\
2 \\
\end{array}$} & $\begin{array}{c}2 \text { - Average } \\
\text { sharpness } \\
0 \\
\end{array}$ & $\begin{array}{c}\begin{array}{c}3 \text { - Below average } \\
\text { sharpness }\end{array} \\
0 \\
\end{array}$ & & & \\
\hline & image & 2 - average sharpness & & 28 & 0 & 30 & & \\
\hline & sharpness - Rl & 3 - below average sharpness & 0 & 1 & 7 & 8 & & \\
\hline \multirow{3}{*}{$\begin{array}{l}\text { Non- } \\
\text { MBIR }\end{array}$} & \multirow{3}{*}{$\begin{array}{l}\text { Subjective } \\
\text { image } \\
\text { sharpness -R1 }\end{array}$} & 1 - above average sharpness & 5 & 0 & 0 & 5 & \multirow[t]{3}{*}{0.817} & \multirow[t]{3}{*}{0.0001} \\
\hline & & 2 - average sharpness & 2 & 31 & 2 & 35 & & \\
\hline & & 3 - below average sharpness & 0 & 0 & 7 & 7 & & \\
\hline
\end{tabular}

MBIR - model-based iterative reconstruction

Our study also showed significant reduction in image noise $(p<0.5)$, whereas the SNR was lower in the MBIR group.

The reduction in radiation doses in MBIR is better compared to adaptive statistical iterative reconstruction (ASIR).
Vorona et al. [8] calculated a $22 \%$ reduction in average $\mathrm{CTDI}_{\mathrm{vol}}$ and a $24 \%$ reduction in average DLP in paediatric head CT, while Kilic et al. [15] calculated a 30\% reduction in average DLP in head CT using ASIR. Olcot et al. [10] 
Table 7. Comparison of the subjective assessment of diagnostic acceptability done by Radiologist 1 and Radiologist 2

\begin{tabular}{|c|c|c|c|c|c|c|c|}
\hline \multicolumn{2}{|l|}{ Group } & \multirow[t]{2}{*}{ Rating } & \multicolumn{2}{|c|}{ Diagnostic acceptability-R2 } & \multirow[t]{2}{*}{ Total } & \multirow[t]{2}{*}{$\kappa$-value } & \multirow[t]{2}{*}{$p$-value } \\
\hline & & & 1 - Fully acceptable & 2-Probably acceptable & & & \\
\hline \multirow[t]{2}{*}{ MBIR } & \multirow[t]{2}{*}{ Diagnostic acceptability - R1 } & 1 - fully acceptable & 38 & 0 & 38 & \multirow[t]{2}{*}{0.788} & \multirow[t]{2}{*}{0.0001} \\
\hline & & 2 - probably acceptable & 1 & 2 & 3 & & \\
\hline \multirow[t]{2}{*}{ Non-MBIR } & \multirow[t]{2}{*}{ Diagnostic acceptability - R1 } & 1 - fully acceptable & 43 & 0 & 43 & \multirow[t]{2}{*}{0.846} & \multirow[t]{2}{*}{0.0001} \\
\hline & & 2 - probably acceptable & 1 & 3 & 4 & & \\
\hline
\end{tabular}

MBIR - model-based iterative reconstruction

Table 8. Comparison of the subjective assessment for artefacts done by Radiologist 1 and Radiologist 2

\begin{tabular}{|c|c|c|c|c|c|c|c|c|}
\hline \multirow[t]{2}{*}{ Group } & & \multirow[t]{2}{*}{ Rating } & \multicolumn{3}{|c|}{ Artefacts-R2 } & \multirow[t]{2}{*}{ Total } & \multirow[t]{2}{*}{$\kappa$-value } & \multirow[t]{2}{*}{$p$-value } \\
\hline & & & 1 - None & 2-Minor & 3-Major & & & \\
\hline \multirow[t]{3}{*}{ MBIR } & \multirow[t]{3}{*}{ Artefacts - R1 } & $1-$ none & 23 & 4 & 0 & 27 & \multirow[t]{3}{*}{0.705} & \multirow[t]{3}{*}{0.0001} \\
\hline & & $2-$ minor & 2 & 10 & 0 & 12 & & \\
\hline & & 3 - major & 0 & 0 & 2 & 2 & & \\
\hline \multirow[t]{3}{*}{ Non MBIR } & \multirow[t]{3}{*}{ Artefacts - R1 } & $1-$ none & 26 & 2 & 0 & 28 & \multirow[t]{3}{*}{0.880} & \multirow[t]{3}{*}{0.068} \\
\hline & & $2-$ minor & 1 & 15 & 0 & 16 & & \\
\hline & & 3 -major & 0 & 0 & 3 & 3 & & \\
\hline
\end{tabular}

MBIR - model-based iterative reconstruction

found that in MBIR, perceived image quality was superior to ASIR and FBP overall $(p<0.001)$. MBIR achieved diagnostic overall perceived image quality with approximately half the radiation dose required by ASIR and FBP. The noise curve of MBIR was significantly lower and flatter $(p<0.001)$. They concluded that MBIR provides superior image quality at half the radiation dose. In our study also, none of the images was found be diagnostically unacceptable on subjective image analysis.

In the study by Katsura et al. [17], 100 adult patients underwent reference-dose and low-dose unenhanced CT scan of the chest. 50\% ASIR-filtered back projection blending (ASIR50) was used for reconstruction of images for reference-dose CT, while both ASIR50 and MBIR were used for reconstruction of images for lowdose CT. The images were evaluated by the 2 radiologists for subjective image noise, artifacts, and diagnostic acceptability. Assessment of objective image noise was done in the lung parenchyma. A 79.0\% decrease in doselength product was seen with low-dose CT when compared to reference-dose CT. This study has determined the possibility of reducing the radiation dose with MBIR compared to ASIR and has again found a significant reduction in the dose.
The present study has some limitations. The scans were done on 2 CT scanners from different manufacturers, which may bias the findings. We did not include specific phantom data to assess radiation dose reduction. Theoretically, the possibility of comparing data from the same individual generated at 2 different points of time (for example, during follow-ups) exists, but this was not our primary objective and not practical due to time constraints. Also, we have measured noise in air, CSF, WM, and GM only. Noise measurements at other sites and at multiple levels could have been obtained to increase the degree of confidence; however, its practical utility and overall statistical difference remain unsubstantiated.

\section{Conclusions}

A model-based iterative reconstruction technique is effective in reducing radiation dose in paediatric head CT examinations with good image quality, image noise, and diagnostic acceptability.

\section{Conflicts of interest}

The authors declare that they have no conflicts of interest. 


\section{References}

1. Sodhi KS, Lee EY. What all physicians should know about the potential radiation risk that computed tomography poses for paediatric patients. Acta Paediatrica 2014; 103: 807-811.

2. Sodhi KS, Krishna S, Saxena AK, Sinha A, Khandelwal N, Lee EY. Clinical application of 'Justification' and 'Optimization' principle of ALARA in pediatric CT imaging: "How many children can be protected from unnecessary radiation?”. Eur J Radiol 2015; 84: 1752-1757.

3. Sodhi KS, Sharma M, Lee EY, et al. Diagnostic utility of 3T lung MRI in children with interstitial lung disease: a prospective pilot study. Acad Radiol 2018; 25: 380-386.

4. Sodhi KS, Khandelwal N, Saxena AK, et al. Rapid lung MRI in children with pulmonary infections: time to change our diagnostic algorithms. J Magn Reson Imaging 2016; 43: 1196-1206.

5. Gunn ML, Kohr JR. State of the art: technologies for computed tomography dose reduction. Emerg Radiol 2009; 17: 209-218.

6. Rogalla P, Kloeters C, Hein PA, et al. CT technology overview: 64-slice and beyond. Radiol Clin North Am 2009; 47: 1-11.

7. Smith B, Dillon WR, Gould R, et al. Radiation dose-reduction strategies for neuroradiology CT protocols. Am J Neuroradiol 2007; 28: 1628-1632.

8. Vorona GA, Zuccoli G, Sutcavage T, et al. The use of adaptive statistical iterative reconstruction in pediatric head CT: a feasibility study. AJNR 2013; 34: 205-211.

9. Ziegler, A, Kohler H, Proksa R, et al. Noise and resolution in images reconstructed with FBP and OSC algorithms for CT. Med Phys 2007; 34: 585-598.

10. Wang G, Yu H, De Man B, et al. An outlook on X-ray CT research and development. Med Phys 2008; 55: 1051-1064.

11. Hajdu SD, Daniel RT, Meuli RA, Zerlauth JB, Dunet V. Impact of model-based iterative reconstruction (MBIR) on image quality in cerebral CT angiography before and after intracranial aneurysm treatment. Eur J Radiol 2018; 102: 109-114.

12. Olcott EW, Shin LK Sommer G, et al. Model-based iterative reconstruction compared to adaptive statistical iterative reconstruction and filtered back-projection in CT of the kidneys and the adjacent retroperitoneum. Acad Radiol 2014; 21: 774-784.

13. Kataria B, Althén JN, Smedby Ö, Persson A, Sökjer H, Sandborg M. Assessment of image quality in abdominal CT: potential dose reduction with model-based iterative reconstruction. Eur Radiol 2018; 28 : 2464-2473.

14. Sun J, Zhang Q, Duan X, et al. Application of a full model-based iterative reconstruction (MBIR) in $80 \mathrm{kVp}$ ultra-low-dose paranasal sinus CT imaging of pediatric patients. Radiol Med 2018; 123: 117-124.

15. Kilic K, Erbas G, Guryildirim M, et al. Lowering the dose in head CT using adaptive statistical iterative reconstruction: Am J Neuroradiol 2011; 32: 1578-1582.

16. Deak PD, Smal Y, Kalender WA, et al. Multisection CT protocols: sex- and age-specific conversion factors used to determine effective dose from dose-length product. Radiology 2010; 257: 158-166.

17. Katsura M, Matsuda I, Akahane M, et al. Model-based iterative reconstruction technique for radiation dose reduction in chest CT: comparison with the adaptive statistical iterative reconstruction technique. Eur Radiol 2012; 8: 1613-1623.

18. Kanal KM, Graves JM, Vavilala MS, Applegate KE, Jarvik JG, Rivara FP. Variation in CT pediatric head examination radiation dose: results from a national survey. Am J Roentgenol 2015; 204: W293-301.

19. Meiville F A, Berteloot L, Jean AG, et al. Model based iterative reconstruction in pediatric chest CT: assessment of image quality in a prospective study of children with cystic fibrosis: Pediatric Radiol 2013; 43: 558-567. 